\title{
AiMT
}

Advances in Military Technology

Vol. 15, No. 2, 2020, pp. 393-403

ISSN 1802-2308, eISSN 2533-4123

DOI 10.3849/aimt.01389

\section{Optimization of Non-uniform Planar Antenna Array Topology in Two-dimensional DOA Estimation}

\author{
X. Luong Tran ${ }^{1 *}$, T. Ha Nguyen ${ }^{1}$, J. Vesely ${ }^{2}$, F. Dvorak ${ }^{2}$, \\ V. Minh Duong ${ }^{2}$ and V. Sang Doan ${ }^{3}$ \\ ${ }^{1}$ Department of Radar Basics, Air Defence, Air Force Academy, Hanoi, Vietnam \\ ${ }^{2}$ University of Defence in Brno, Czech Republic \\ ${ }^{3}$ Department of Radar, Naval Academy, Nhatrang, Vietnam
}

The manuscript was received on 16 February 2020 and was accepted after revision for publication as research paper on 2 November 2020.

\begin{abstract}
:
The paper deals with the optimization of the sparse planar antenna array for direction of arrival (DOA) estimation in two dimensions (azimuth and elevation). The optimization algorithm was proposed on the basis of the peak sidelobe level (SLL) and half-power beamwidth $(H P B W)$ parameters. In empirical validation, we have set up a measurement system to test the efficiency of an optimized array configuration. The array was configured using the patch antennas with frequency band $2.4 \mathrm{GHz}$. In comparison with several popular array configurations, the experimental results show that the proposed antenna array was optimized, and it provided higher accuracy and resolution in two-dimensional (2D) DOA estimation.
\end{abstract}

\section{Keywords:}

direction of arrival estimation, optimization, sparse planar array, 2D MUSIC algorithm

\section{Introduction}

Direction of arrival (DOA) estimation refers to the process of finding the direction information of the electromagnetic or acoustic waves, which are impinging on a sensor or an antenna array [1]. The DOA estimation has been utilized for locating and tracking signal sources in both civilian and military applications, such as search and rescue, radar and sonar systems, and seismology [2].

In practice, the DOA estimation techniques are usually applied for the uniform and non-sparse arrays, such as uniform linear arrays (ULAs), uniform rectangular arrays (URAs) and uniform L-shaped arrays [3-5]. They require that the distance between the two neighbouring antenna elements is not greater than half the wavelength

\footnotetext{
* Corresponding author: Department of Radar Basics, Air Defence, Air Force Academy, Hanoi, Vietnam.E-mail: xuanluong.tran90@gmail.com
} 
of impinging signals. Recently, for obtaining more accurate direction information, the high-resolution subspace algorithms have been researched and developed [1].

In communication systems, two-dimensional (azimuth and elevation) DOA estimation also plays an important role within array signal processing. Estimating of the azimuth and elevation angles provides more information about the direction and location of the incoming sources. Therefore, the performance of the communication systems is improved. By composing two perpendicular uniform linear arrays, an L-shaped or cross-shaped array is created to determine azimuth and elevation angles, simultaneously. In spite of 2D DOA sufficient estimation capability, those two particular configurations have the disadvantage of limited array aperture, which leads to decreasing the accuracy in DOA determination [4-7].

In many applications, such as radar, sonar, communication, and astronomy, the DOA estimation accuracy can be enhanced by using antenna arrays with a large aperture, which can be designed by multiple antenna elements in uniform distances. However, uniform linear arrays require expensive hardware and computational complexity. In order to reduce the hardware cost and computational burden, non-uniform (or sparse) arrays can be cleverly designed to yield the same performance as ULA in case of the same aperture or to gain higher accuracy than the ULA with the same number of elements. Therefore, designing an optimal non-uniform array that can improve the DOA estimation accuracy and also reduce the element number is significant [8-11].

In other researches, different approaches had been presented to optimize the element positions in the non-uniform linear array for DOA estimation of a single incoming signal. These methods were proposed based on the minimization of CramerRao bound (CRB), and Weiss-Weinstein bound (WWB) [12, 13]. Also, a non-uniform linear array could be optimized based on the parameters of peak sidelobe level (SLL) and half-power beamwidth (HPBW or $\theta_{-3 \mathrm{~dB}}$ ). This optimization algorithm has reached better performance in DOA estimation for the case of multiple incoming signals [14].

While the issue is considerably solved in the 1D case, the question of optimal antenna placement in the 2D arrays is more complicated, and it has been addressed in various ways, mostly employing optimization techniques based on evolutionary concepts.

In this research work, we have considered the case of the non-uniform planar array with integer element spacing, a fixed aperture, and a fixed number of antenna elements. The considered array was designed and applied for various effective DOA algorithms, such as spectral MUSIC or Root-MUSIC. It was assumed that the number of incoming signals was known. Therefore, the optimized antenna array mainly relied on SLL and HPBW. Then, the optimized array configuration was tested to evaluate the DOA estimation accuracy for the case of multiple source signals.

The structure of this paper was organized as follows: The signal model and 2D MUSIC algorithm are presented in Section 2. In Section 3, an optimization algorithm for the non-uniform planar array is proposed. The measurement results are presented in Section 4, where the different 2D antenna configurations with the different number of elements are tested. Finally, the main conclusions drawn from the measurement results are summarized in Section 5. 


\section{Signal Model and MUSIC Algorithm}

\subsection{Signal Model}

In order to estimate the two-dimensional DOA of signals, we considered a nonuniform planar array with $M$ identical omnidirectional antenna elements, as shown in Fig. 1. The inter-element spacing between the two antenna elements is $d$ in both the $x$ axis and $y$-axis.

Let us assume that there were $K$ far-field sources which radiated uncorrelated narrowband signals with wavelength $\lambda$ that were incoming on the considered array from $K$ directions at angles $\theta_{\mathrm{i}}$ in azimuth and $\phi_{\mathrm{i}}$ in elevation. Each signal received by the array was known as $\boldsymbol{x}(t)$ including additive zero-mean Gaussian noise. In matrix form, the output of the array could be given in the following form [1]:

$$
\boldsymbol{x}(t)=\boldsymbol{A}(\theta, \phi) \boldsymbol{s}(t)+\boldsymbol{n}(t)
$$

where $\boldsymbol{x}(t)$ was the $M \times 1$ snapshot vector of the signals received simultaneously on all the antennas; $\mathbf{s}(t)$ was the $K \times 1$ vector of the source signals; $\boldsymbol{n}(t)$ was the $M \times 1$ noise vector; the $M \times K$ steering matrix $\boldsymbol{A}(\theta, \phi)=\left[\boldsymbol{a}\left(\theta_{1}, \phi_{1}\right), \ldots, \boldsymbol{a}\left(\theta_{M}, \phi_{M}\right)\right]$ was defined as the array manifold and consisted of the steering vector $\boldsymbol{a}\left(\theta_{k}, \phi_{k}\right)$ whose components were:

$$
\boldsymbol{a}_{m}\left(\theta_{k}, \phi_{k}\right)=\mathrm{e}^{\mathrm{j} 2 \pi f \cdot \tau_{m}\left(\theta_{k}, \phi_{k}\right)}
$$

where $\tau_{m}\left(\theta_{k}, \phi_{k}\right)=d_{k}^{\mathrm{T}}\left(\theta_{k}, \phi_{k}\right) \frac{\boldsymbol{r}_{m}}{c}$ was the propagation delay of the received signal from reference array element to the $m$-th array element for a $k$-th incoming signal $(1 \leq m \leq M) ; c$ was the speed of electromagnetic wave propagation in the medium and $\boldsymbol{d}_{k}\left(\theta_{k}, \phi_{k}\right)$ was the unit vector pointing toward $k$-th source $(1 \leq k \leq K)$.

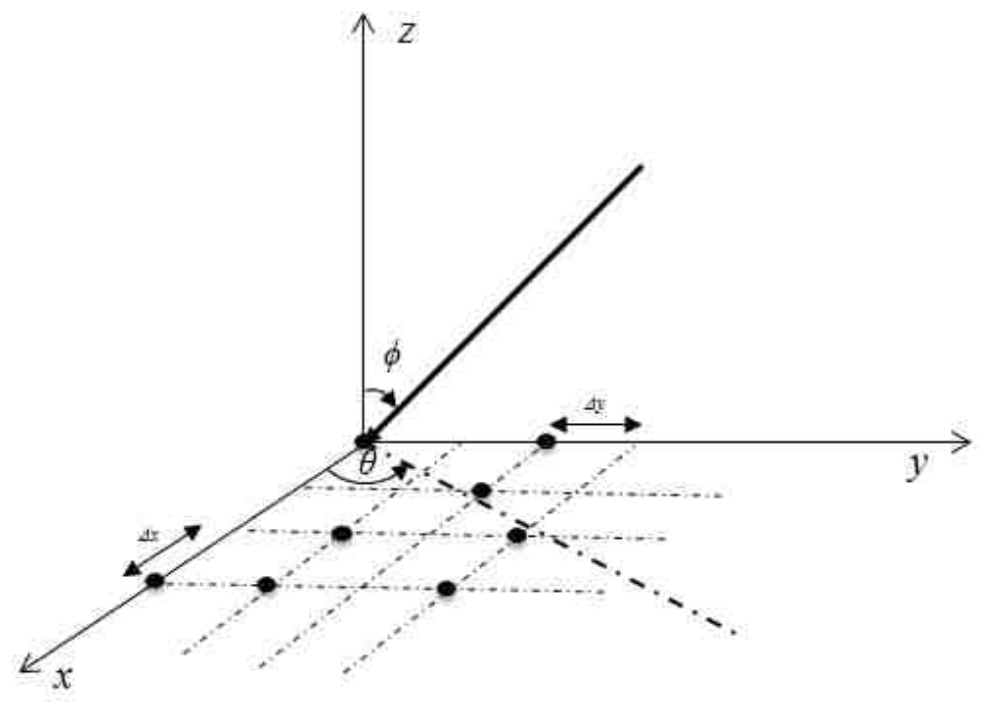

Fig. 1 Geometry of non-uniform planar antenna array with $M=8$ elements 


$$
d[\theta, \phi]=[\cos \phi \cos \theta, \sin \phi \sin \theta]
$$

$\boldsymbol{r}_{m}=\left[x_{m}, y_{m}\right]^{\mathrm{T}}$ was the position vector of $m$-th element that depends on the geometry of the antenna array. In this paper, the non-uniform planar array would be addressed. The propagation delay for the $k$-th source in the $m$-th element was derived as

$$
\boldsymbol{\tau}_{m}\left[\theta_{k}, \phi_{k}\right]=\frac{\left(x_{m} \cos \theta_{k}+y_{m} \sin \theta_{k}\right) \sin \phi_{k}}{c}
$$

The purpose of DOA estimation is to determine the indication function of arrival angle. This function was called the pseudo spectrum $P(\theta, \phi)$ and we used the standard 2D MUSIC algorithm as high-resolution eigen-structure method for direction finding purpose.

\subsection{MUSIC Algorithm}

For an explanation of MUSIC algorithm, a non-uniform array was considered in case of uncorrelated noise with equal variance $\delta$. The covariance matrix $\boldsymbol{R}_{x x}$ was defined as follows

$$
\boldsymbol{R}_{x x}=\mathrm{E}\left[\boldsymbol{x}(t) \boldsymbol{x}^{\mathrm{H}}(t)\right]
$$

where []$^{\mathrm{H}}$ represented a conjugate transposition. In practice, the array covariance matrix is estimated from available data, $\hat{\boldsymbol{R}}$ based on a finite number of data samples $(P)$ or snapshot.

$$
\hat{\boldsymbol{R}}=\frac{1}{P} \sum_{j=1}^{P} \boldsymbol{x}\left(t_{j}\right) \boldsymbol{x}^{\mathrm{H}}\left(t_{j}\right)
$$

MUSIC algorithm relied on the eigen decomposition of the covariance matrix $\hat{\boldsymbol{R}}=\boldsymbol{V} \cdot \boldsymbol{\Lambda} \cdot \boldsymbol{V}^{\mathrm{H}}$, where $\boldsymbol{V}=\left[v_{1}, v_{2}, \ldots, v_{M}\right]$. was the eigenvector corresponding with the eigenvalue $\boldsymbol{\Lambda}=\operatorname{diag}\left[\lambda_{1}, \lambda_{2}, \ldots, \lambda_{M}\right] ; \boldsymbol{v}_{K}$ is the subspace eigenvector corresponding with the eigenvalue $\lambda_{K}$. The eigenvalues were sorted as $\lambda_{1}>\lambda_{2}>\ldots>\lambda_{M}$.

If the number of sources $K$ was smaller than the number of antenna elements $M$, all signal components would be presented in the signal subspaces, spanned by the first $K$ eigenvectors $v_{1}, v_{2}, \ldots, v_{K}$ which are representing the noise subspaces. The subspaces of the signals and the noise are orthogonal. The subspace spanned by the $K$ steering vectors.

Generally, we applied the 2D MUSIC algorithm to find the azimuth and elevation angle of signals, the spatial spectrum function of signals was given by

$$
P(\theta, \phi)=\frac{\boldsymbol{a}^{\mathrm{H}}(\theta, \phi) \boldsymbol{a}(\theta, \phi)}{\boldsymbol{a}^{\mathrm{H}}(\theta, \phi) \boldsymbol{E}_{n} \boldsymbol{E}_{n}^{\mathrm{H}} \boldsymbol{a}(\theta, \phi)}
$$

where $\boldsymbol{E}_{n}$ was noise subspaces and computed as $\boldsymbol{E}_{n}=\left[v_{1}, v_{2}, \ldots, v_{K}\right]$.

When the subspaces were determined, the angles of arrival of desired signals could be estimated by finding the maxima of the MUSIC spatial spectrum. The 2D MUSIC algorithm created a two-dimensional grid, in the range which the angles vary $\theta \in\left[0,360^{\circ}\right]$ and $\phi \in\left[0,90^{\circ}\right]$. 


\section{Optimization of Antenna Element Positions}

In this section, we focused on optimizing the element positions in the antenna array with an aperture array size $B \times B$, where $B \in \mathbb{N}$ For $1 \mathrm{D}$ antenna array, the optimum geometry configuration was solved by minimizing the CRB and WWB for multiincoming signal could be used. However, these approaches were quite difficult and impractical for a $2 \mathrm{D}$ antenna array due to computational complexity. The goal of the 2D array geometry optimization was to identify geometries with minimum SLL with acceptable $\phi_{\text {threshold. }}$

In this paper, non-uniform planar arrays were presented with integer element spacings, fixed array apertures and fixed numbers of elements. Therefore, these arrays could be applied directly using the spectral 2D MUSIC algorithm. For simplicity, antenna elements were assumed to be put into a normalized grid of integers contained within a rectangle with size of $B \times B$. The first element was in the origin $O(0,0)$. The challenge of optimization was finding the optimal element positions $(x, y)$ in the range of $(0, B)$.

The proposed optimization algorithm of the element positions was based on the theory of the antenna array, associated directly to the SLL and HPBW. The array beam pattern was defined by

$$
B(\theta, \phi)=\sum_{m=1}^{M} \mathrm{e}^{\mathrm{j} \frac{2 \pi}{\lambda} x_{m} \sin \phi \cos \theta+y_{m} \sin \phi \sin \theta}
$$

The normalized array beam pattern was defined by:

$$
G(\theta, \phi)=\frac{|B(\theta, \phi)|^{2}}{M^{2}}
$$

We may write the corresponding optimization problem in the form

$$
\begin{aligned}
\min \left\{\max _{\phi}\left[\operatorname{SLL}\left(x_{1}, y_{1}, x_{2}, \ldots, y_{M}\right)\right]\right\} \\
\text { s.t. }\left\{\begin{array}{l}
x_{1}=0, y_{1}=0 \\
0 \leq x_{2}, y_{2}, \ldots, y_{M} \leq B \\
x_{i} ; y_{i} \in \mathbb{N}, i=2, \ldots, M \\
\theta \in\left[0,180^{\circ}\right] \\
\phi \in\left[\phi_{\text {threhold }}, 90^{\circ}\right]
\end{array}\right.
\end{aligned}
$$

Here, the objective function was multimodal function with respect to the element position. The optimization problem was to find minimum SLL in the limited side lobe region $\left[\phi_{\text {threshold }}, 90^{\circ}\right.$. The parameter $\phi_{\text {threshold }}$ could be derived from the equation calculated HPBW of the rectangular planar array and it was calculated as $\phi_{\text {threshold }}=2 / B$ [rad].

Therefore, the Genetic Algorithm could be used to solve these optimization problems. In the MATLAB, function ga() was used for solving the optimization problem, which was set up with default parameters [15]. We considered optimum planar arrays of 8 elements, which were positioned in a square with a size of $7 \times 7$. The desired signal was assumed to be incoming from the azimuth of $\left[0,360^{\circ}\right]$ and the elevation of $\left[0,90^{\circ}\right]$. The resulting antenna array from solving the optimization problem is shown in Fig. 2. 


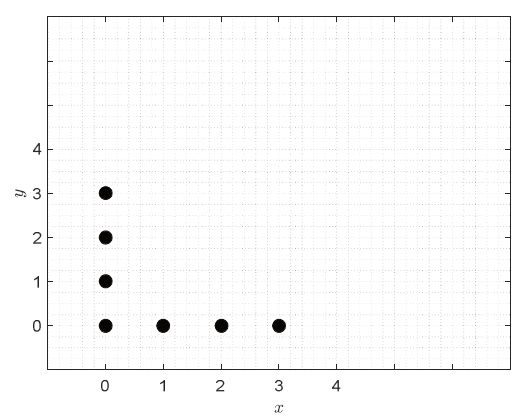

(a) Configuration $\mathrm{A}$

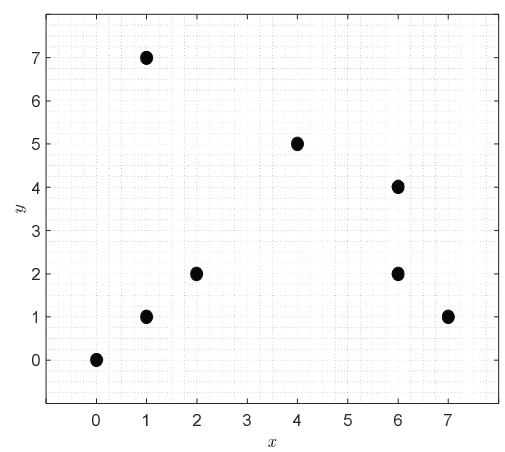

(c) Configuration $\mathrm{C}$

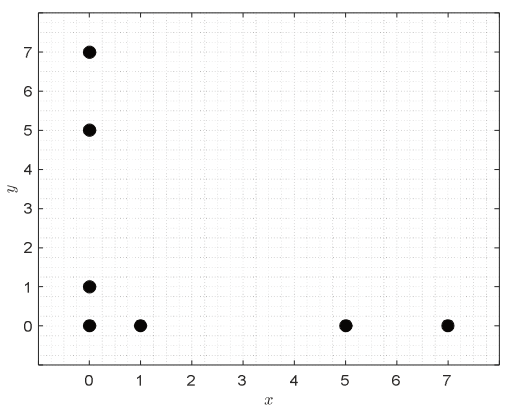

(b) Configuration $\mathrm{B}$

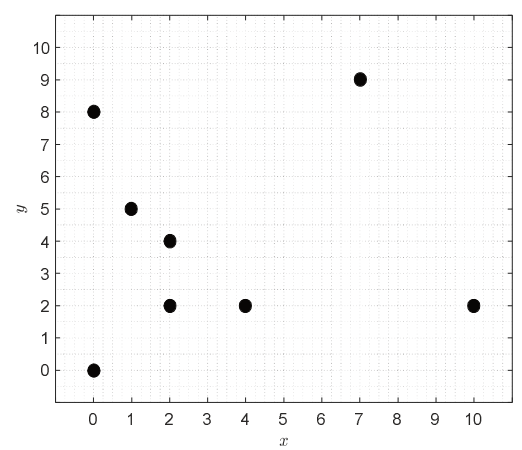

(d) Configuration D

Fig. 2 (a) Standard uniform L-sharp array; (b) Non-uniform L-sharp array;

(c) Optimal non-uniform array with size of $7 \times 7$ and $S L L=-4.54 \mathrm{~dB}$;

(d) Optimal non-uniform array with size of $10 \times 10$ and $S L L=-3.14 \mathrm{~dB}$

\section{Measurement results}

In this section, we evaluated the performance of the optimal configuration for DOA estimation in comparison with existing configurations in case of the same aperture or number of elements. The practical measurements were carried out in the microwave anechoic chamber.

The receiving antenna arrays were composed of microstrip patch antennas, which were designed for central operating frequency $f=2.45 \mathrm{GHz}$. The antennas were fabricated on the PTFE/Teflon substrate with relative permittivity $\varepsilon_{r}=2.1$, and the loss tangent equals 0.001 at the frequency of $2.45 \mathrm{GHz}$ [16]. Outputs of the antenna elements were connected to the inputs of 4-channel R\&S RTO 1014 oscilloscope via coaxial cables with the same electric length as shown in Fig. 3.

The implemented system consists of an antenna array, two four-channel oscilloscopes, and impulse signal generator for synchronization (output trigger). The logged data displayed on the oscilloscopes were saved to *.csv files for further signal processing in a computer (PC). Then, the DOA estimation algorithm was performed in MATLAB with the saved data in the PC. The antenna elements were spaced on the grid with $d=d x=d y=0.061 \mathrm{~m}$. 


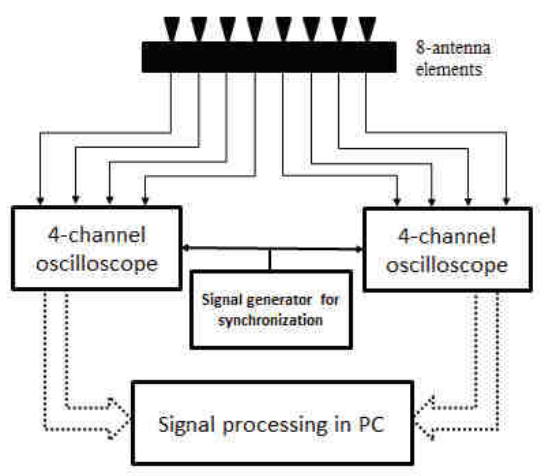

(a)

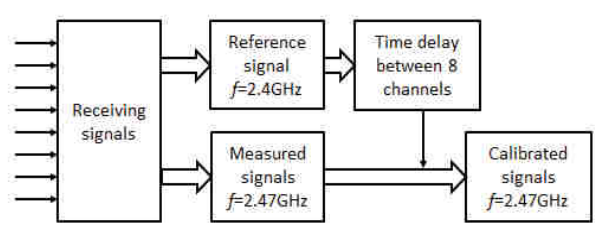

(b)

Fig. 3 (a) Block diagram of testing equipment for 8-element arrays,

(b) Calibration process of measured signals

In the first experiment, we compared the 2D DOA estimation results of four kinds of array configurations for one incoming signal source. The first configuration, namely configuration A (Fig. 2a), was a uniform L-sharp array with the aperture $3 \times 3$. The second configuration, namely configuration B (Fig. 2b), was a non-uniform Lsharp array, which is composed of two non-uniform linear arrays with the aperture $7 \times 7$. The third configuration, namely configuration $C$ (Fig. 2 c), was an optimal array that was derived from the optimization algorithm according to Section 3, with SLL $=-4.54 \mathrm{~dB}$. The last configuration, namely configuration D (Fig. 2d), was an array with aperture $10 \times 10$ and SLL $=-3.14 \mathrm{~dB}$.

For the DOA estimation, two types of transmitting antennas were used: (1) a monopole antenna (yellow circle) is referent to calibrate; (2) a Vivaldi antenna (red circle) was used for testing. The algorithm to calibrate the receiving signals is shown in Fig. 3b. The arrangement of the transmitting antennas in the anechoic chamber is shown in Fig. 4b.

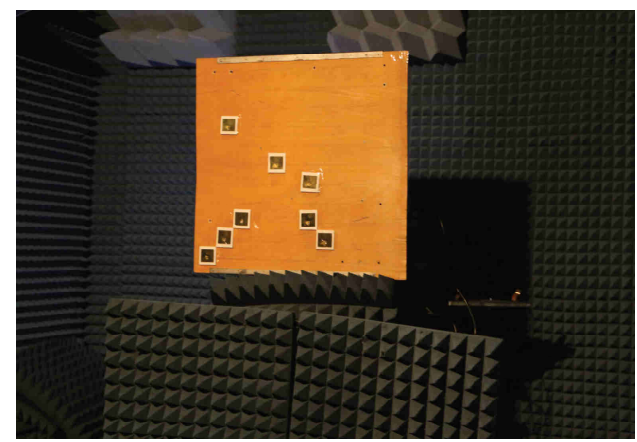

(a)

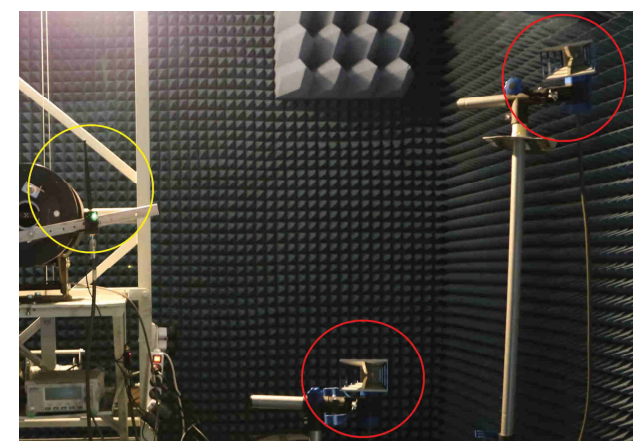

(b)

Fig. 4 (a) Receiving antenna array; (b) Transmitting antennas in anechoic chamber

In our measurement, the reference signal generator was located at the origin position $\left(\theta_{0}, \phi_{0}\right)=\left(0^{\circ}, 0^{\circ}\right)$, and the measured signal generator was located at the direction $(\theta, \phi)=\left(15^{\circ}, 24^{\circ}\right)$. 
The 2D MUSIC spectra of 4 arrays with various configurations for DOA estimation of one incoming signal source is shown in Fig. 5. In order to simplify determining and extracting peaks of 2D MUSIC spectra, a normalization function to detect correctly the number of maximum values was implemented or the algorithm in [17] by Natan could be directly used. The results of peak finding shown in Fig. 5 indicated one highest peak (black square). The DOA estimation results of four array configurations are obtained, and it was obvious that all configurations could be used to estimate successfully DOA of one incoming signal source; the estimated directions were quite close to the initially proposed directions. The root mean square error (RMSE) was selected to evaluate the DOA estimation accuracy of the different configuration:

$$
R M S E=\frac{1}{K} \sum_{k=1}^{K} \sqrt{\frac{1}{N} \sum_{n=1}^{N}\left(\theta_{k, n}-\theta_{k, 0}\right)^{2}+\left(\phi_{k, n}-\phi_{k, 0}\right)^{2}}
$$

where $K$ is the number of incoming signal sources, $N$ is the number of experiment trials, $\theta_{k, n}, \phi_{k, n}$, is the estimated azimuth and elevation $k$-th signal source for the $n$-th trial, $\theta_{k, 0}, \phi_{k, 0}$, is the proposed azimuth an elevation.

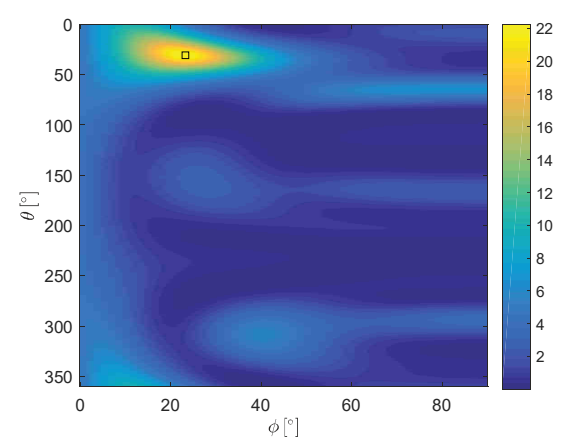

(a)

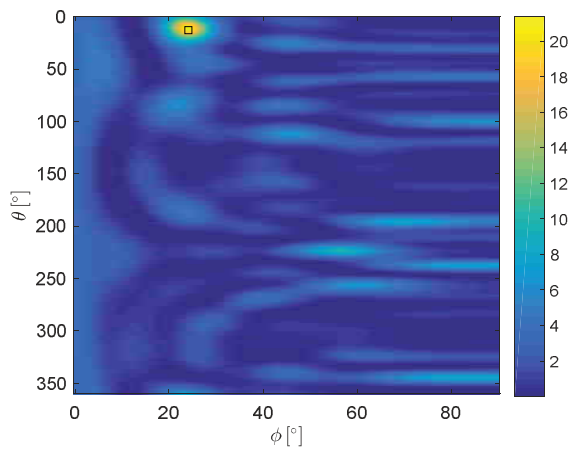

(c)

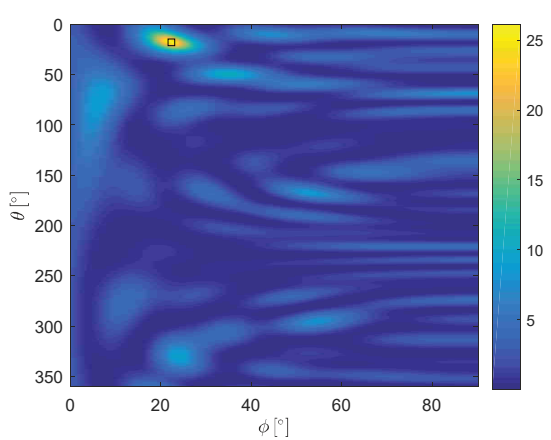

(b)

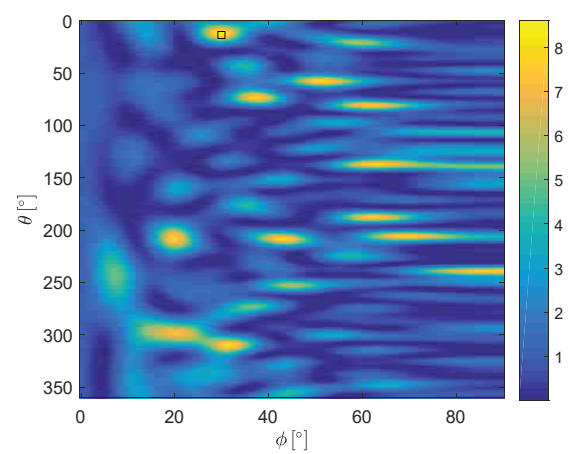

(d)

Fig. 5 Two-dimensional MUSIC spectra of 4 configurations

The above results have shown that antenna array configurations presented in Figs $2 \mathrm{a}, 2 \mathrm{~b}$ and $2 \mathrm{c}$ had better estimation performance than the configuration presented in Fig. 2d, which appeared more ambiguous lobes in the 2D MUSIC spectrum. Therefore, we conducted $\mathrm{N}=10$ experiment trials to validate the estimation performance of the three first array configurations. The RMSE of three array configurations have been 
obtained: configuration A had RMSE $=8.49^{\circ}$; configuration $\mathrm{B}$ had RMSE $=6.26^{\circ}$ and configuration $\mathrm{C}$ had $\mathrm{RMSE}=2.28^{\circ}$. It was clear that the optimal configuration $\mathrm{C}$ gained higher accuracy than two others in DOA estimation.

In the second experiment, we compared the 2D DOA estimation results of three array configurations for signal sources incoming from two close angles. Their incoming directions were $\left(\theta_{1}, \phi_{1}\right)=\left(10^{\circ}, 11^{\circ}\right)$ and $\left(\theta_{2}, \phi_{2}\right)=\left(14^{\circ}, 20^{\circ}\right)$, respectively. The 2-D MUSIC spectra for the DOA estimation of two signal sources shown in Fig. 6 indicated that the optimal array configuration $\mathrm{C}$ allowed to distinguish the two peaks of spectrum which are corresponding to two measured directions of the signal sources. For the uniform L-sharp and non-uniform L-sharp antenna array configurations, the two peaks were not clearly distinguished.

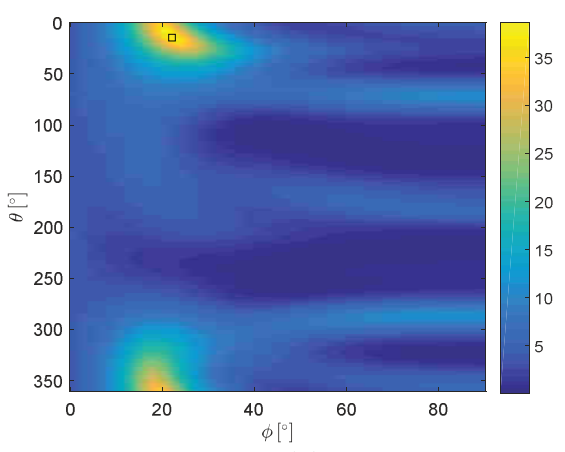

(a)

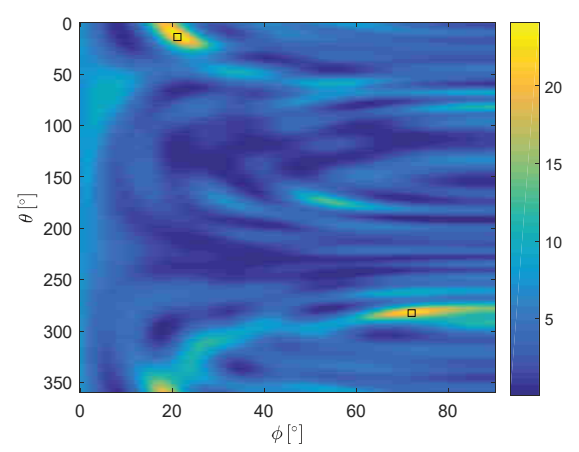

(b)

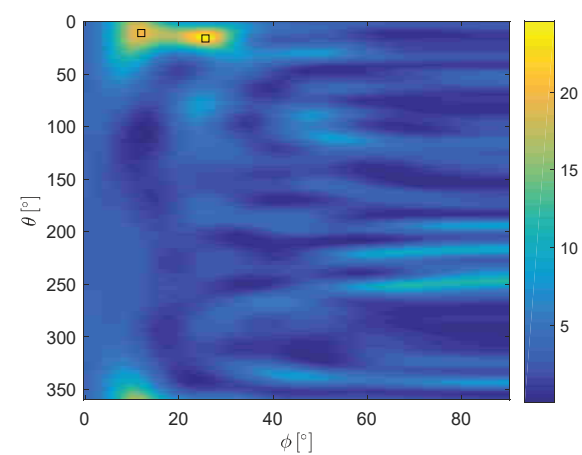

(c)

Fig. 6 Two-dimensional MUSIC spectra of two close signal sources with antenna array a) configuration $A, b$ ) configuration $B$ and c) configuration $C$

Finally, we presented the DOA estimation result of the optimal antenna array configuration for three incoming signal sources. The directions of three signal sources were $\left(\theta_{1}, \phi_{1}\right)=\left(10^{\circ}, 15^{\circ}\right),\left(\theta_{2}, \phi_{2}\right)=\left(165^{\circ}, 1^{\circ}\right)$ and $\left(\theta_{3}, \phi_{3}\right)=\left(285^{\circ}, 17^{\circ}\right)$, respectively. Fig. 7 showed that our optimal configuration (Fig. 2c) has significantly better resolution and DOA performance than other configurations.

\section{Conclusion}

In this paper, an algorithm to optimize element positions of $2 \mathrm{D}$ non-uniform planar array has been proposed. The optimal array configuration was argued based on SLL 


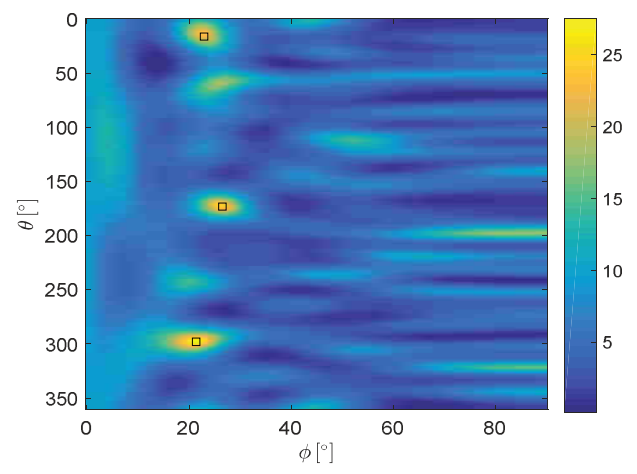

Fig. 7 Two-dimensional MUSIC spectrum

and HPBW. The optimized array configuration was evaluated by experimental measurement, and then it was compared with several other popular configurations in the case of the same aperture and number of antenna elements. The experiment results showed that our optimized array configuration reached better performance with higher accuracy and resolution of DOA estimation than other mentioned configurations. However, these optimized arrays only estimate correctly when the number of signals did not exceed the number of array elements $(K<M)$. In future works, we will com bine this optimization algorithm with other optimization criteria to obtain a greater number of signal sources and performance in DOA estimation.

\section{Acknowledgement}

This work has been supported by the project for development of K-207 department, University of Defence in Brno, Ministry of Defence of the Czech Republic - Research of sensor and control systems to achieve battlefield information superiority.

\section{References}

[1] CHANDRAN, S. Advances in Direction-of-Arrival Estimation. Boston: Artech House, 2005. 496 p. ISBN 978-1-59-693004-9.

[2] HARRY, L. van T. Optimum Array Processing: Part IV of Detection, Estimation, and Modulation Theory. New York: Wiley, 2002. 1433 p. ISBN 978-0-47109390-9.

[3] KRIM, H. and VIBERG, M. Two Decades of Array Signal Processing Research: the Parametric Approach. IEEE Signal Processing Magazine, 1996, vol. 13, no. 4, p. 67-94. DOI 10.1109/79.526899.

[4] BALANIS, C.A. Antenna Theory Analysis and Design. $4^{\text {th }}$ ed. New York: Wiley, 2016. 1104 p. ISBN 978-1-118-64206-1.

[5] HEIDENREICH, P., ZOUBIR, A.M. and RUBSAMEN, M. Joint 2-D DOA Estimation and Phase Calibration for Uniform Rectangular Arrays. IEEE Transactions on Signal Processing, 2012, vol. 60, no. 9, p. 4683-4693. DOI 10.1109/TSP.2012.2203125. 
[6] GU, F., ZHU, W.P. and SWAMY, M.N.S. Joint 2-D DOA Estimation Viasparse L-Shaped Array. IEEE Transactions on Signal Processing, 2015, vol. 63, no. 5, p. 1171-1182. DOI 10.1109/TSP.2015.2389762.

[7] CHEN, H., HOU, C., WANG, Q., HUANG, L., YAN, W. and PU, L. Improved Azimuth/Elevation Angle Estimation Algorithm for Three-Parallel Uniform Linear Arrays. IEEE Antennas and Wireless Propagation Letters, 2015, vol. 14, p. 329-332. DOI 10.1109/LAWP.2014.2360419.

[8] TAYEM, N. and KWON, H. L-Shape 2-Dimensional Arrival Angle Estimation with Propagator Method. IEEE Transactions on Antennas and Propagation, 2005, vol. 53, no. 5, p. 1622-1630. DOI 10.1109/TAP.2005.846804.

[9] VIBERG, M. and ENGDAHL, C. Element Position Considerations for Robust Direction Finding Using Sparse Arrays. In Proceedings of the Thirty-Third Asilomar Conference on Signals, Systems, and Computers. Pacific Grove: IEEE, 1999, p. 835-839. DOI 10.1109/ACSSC.1999.831827.

[10] LANGE, O. and YANG, B. Antenna Geometry Optimization for 2D DirectionOf-Arrival Estimation for Radar Imaging. In Proceedings of the International ITG Workshop on Smart Antennas. Aachen: IEEE, 2011, p. 6-13. DOI 10.1109/WSA.2011.5741909.

[11] COHEN, R. and ELDAR, Y.C. Sparse Convolutional Beamforming for Ultrasound Imaging. IEEE Transactions on Ultrasonics, Ferroelectrics, and Frequency Control, 2018, vol. 65, no. 12, p. 2390-2406. DOI 10.1109/TUFFC.2018.2874256.

[12] ATHLEY, F. Optimization of Element Positions for Direction Finding with Sparse Arrays. In Proceedings of the $11^{\text {th }}$ IEEE Signal Processing Workshop on Statistical Signal Processing. Singapore: IEEE, 2001, p. 516-519. DOI 10.1109/SSP.2001.955336.

[13] WANG, X., ABOUTANIOS, E. and AMIN, M.G. Adaptive Array Thinning for Enhanced DOA Estimation. IEEE Signal Processing Letters, 2015, vol. 22, no. 7, p. 799-803. DOI 10.1109/LSP.2014.2370632.

[14] TRAN, X.L., VESELY, J., HUBACEK, P. and DUONG, V.M. Optimization of Nonuniform Linear Array Configuration for Reduced Number of Elements. In Proceedings of the $19^{\text {th }}$ International Radar Symposium. Bonn: IEEE, 2018, p. 1057-1066. DOI 10.23919/IRS.2018.8448063.

[15] Find Minimum of Function Using Genetic Algorithm [online]. [viewed 2020-0115]. Available from: https://www.mathworks.com/help/gads/ga.html

[16] TRAN, X.L., VESELY, J. and DVORAK, F. Optimization of Nonuniform Linear Antenna Array Topology. Advances in Electrical and Electronic Engineering, 2018, vol. 16, no. 3, p. 341-349. DOI 10.15598/aeee.v16i3.2841.

[17] Fast 2D Peak Finder [online]. October 2013. [viewed 2020-01-09]. Available from: https://www.mathworks.com/matlabcentral/fileexchange/37388-fast-2dpeak-finder 